\title{
Identification of intra- and inter-individual metabolite variation in plasma metabolite profiles of cats and dogs
}

\author{
Alison Colyer ${ }^{1}$, Matthew S. Gilham ${ }^{1}$, Beate Kamlage ${ }^{2}$, Dietrich Rein ${ }^{3}$ and David Allaway ${ }^{1 *}$ \\ ${ }^{1}$ Waltham Centre for Pet Nutrition, Freeby Lane, Waltham-on-the-Wolds, Leicestershire LE14 4RT, UK \\ ${ }^{2}$ Metanomics GmbH, Tegeler Weg 33, 10589 Berlin, Germany \\ ${ }^{3}$ Metanomics Health GmbH, Tegeler Weg 33, 10589 Berlin, Germany \\ (Received 13 October 2010 - Revised 13 January 2011 - Accepted 28 January 2011)
}

\begin{abstract}
The purpose of the present study was first to identify drivers of variance in plasma metabolite profiles of cats and dogs that may affect the interpretation of nutritional metabolomic studies. A total of fourteen cats and fourteen dogs housed in environmentally enriched accommodation were fed a single batch of diet to maintain body weight. Fasting blood samples were taken on days 14,16 and 18 of the study. Gas chromatography-mass spectrometry (GC-MS), liquid chromatography (LC)-MS/MS and solid-phase extraction-LC-MS/MS analyses were used for metabolite profiling. Principal component (PC) analysis that indicated 31 and $27 \%$ of the variance was explained in PC1 and PC2 for cats and dogs, respectively, with most individuals occupying a unique space. As the individual was a major driver of variance in the plasma metabolome, the second objective was to identify metabolites associated with the individual variation observed. The proportion of intra- and inter-individual variance was calculated for 109 cat and $101 \mathrm{dog}$ metabolites with a low intra-individual variance (SD $<0.05$ ). Of these, fifteen cat and six dog metabolites had inter-individual variance accounting for at least $90 \%$ of the total variance. There were four metabolites common to both species (campesterol, DHA, a cholestenol and a sphingosine moiety). Many of the metabolites with $>75 \%$ inter-individual variance were common to both species and to similar areas of metabolism. In summary, the individual is an important driver of variance in the fasted plasma metabolome, and specific metabolites and areas of metabolism may be differentially regulated by individuals in two companion animal species.
\end{abstract}

Key words: Cats: Dogs: Plasma metabolites: Nutritional metabolomics

Metabolomics may be defined as the measure of as many metabolites as possible in a biological sample. It has many applications such as identifying potential diagnostic biomarkers in disease pathology, establishing drug modes of action and investigating biological phenomena through datadriven hypothesis generation. A strength of the data-driven approach is that it enables investigations of complex, nonlinear, interactive multivariate systems which are difficult to control or where no clear hypothesis exists ${ }^{(1)}$. Nutrition is a multi-factorial and complex process, and nutritional metabolomics has been proposed as a powerful tool to investigate the interaction between the individual and their $\operatorname{diet}^{(2)}$, and the influence and roles of the individual, their genetics, lifestyle and gastrointestinal flora have been assessed ${ }^{(3)}$.

In the USA and European Union, people are responsible for providing, at least in part, nutritional requirements of more than 250 million companion animals ${ }^{(4,5)}$, and responsible pet food manufacturers undertake research to ensure their products are nutritionally complete and safe. However, the role of the individual animal in determining their nutritional needs is yet to be elucidated. It is possible that the range of physiologies and genetic backgrounds in dogs makes size and breed important parameters to consider. However, in cats, the more limited physiological variation may have a less impact on the individual needs. Previously, NMR analysis of urine from dogs fed the same diet in the same environment identified that metabolite profiles were indicative of the individual and the breed ${ }^{(6)}$. Characterisation of urinary metabolites by flow injection electrospray-MS and GC-MS suggests a difference between breeds in their digestion of complex plant materials, while variation in the urinary metabolome of client-owned dogs indicated that breed effects may be confounded by environmental factors ${ }^{(7)}$. These observations indicate that to allow accurate interpretation of nutritional metabolomics data, study designs need to factor the major drivers of variance in the metabolome and to identify those

Abbreviations: DHA, docosahexaenoic acid; GC, gas chromatography; LC, liquid chromatography; MS, mass spectrometry; PC, principal component; PCA, principal component analysis.

*Corresponding author: D. Allaway, fax + 441664 415440, email david.allaway@effem.com 
metabolites that are most sensitive to these drivers. The initial objective of the present study was to identify the drivers of variance in plasma metabolite profiles of cats and dogs when fed the same diet in an environmentally controlled nutritional metabolomics study. The second objective was to identify those metabolites that correlated with any major drivers of variance.

\section{Experimental methods}

\section{Study design}

Labrador retriever dogs (fourteen spayed females, aged 1-9 (mean 6.2, SD 3.0)) years and domestic short-haired cats (fourteen spayed females, aged 1-9 (mean 5.0, SD 1.6)) years were randomly assigned to two groups that were fed 4 weeks apart (seven animals per group). Blood samples were obtained from each animal on days 14, 16 and 18 of the study.

\section{Animal maintenance and metadata collection}

Animals were housed in purpose-built, environmentally enriched facilities at the Waltham Centre for Pet Nutrition (Waltham-on-the-Wolds, Leicestershire, UK), in accordance with the Centre's research ethics and UK Home Office Regulations. They had free access to water and were maintained on a single batch of Whiskas Chicken in Jelly cat food ( $100 \mathrm{~g}$ pouch; Mars Petcare UK Limited, Melton Mowbray, UK) throughout the study at energy levels to maintain adult body weight ( $\pm 10 \%$ ideal body weight). The average weight of dogs was 24.7 (SD 1.9) kg, and the average weight of cats was 4.5 (SD 0.4) kg.

Diet was provided between 08.00 and 09.00 hours daily, and the amount of diet offered and percentage eaten were recorded. Additional information was also available (e.g. age, years spent neutered, body weights and body condition scores, faecal quality, pre-trial diet, sibling group, times of sampling, centrifugation and freezing).

\section{Plasma preparation}

After fasting $(23 \mathrm{~h})$, blood was collected into EDTA tubes (from the cephalic vein in dogs $(10 \mathrm{ml}$ ) and from the jugular vein in cats $(5 \mathrm{ml})$ ). The tubes were inverted and incubated on ice (maximum $30 \mathrm{~min}$ ) before centrifugation (1000 $\mathrm{g}$, $10 \mathrm{~min}$ at $4^{\circ} \mathrm{C}$ ). Plasma supernatants (forty-two dogs and forty-one cats) were stored $\left(-80^{\circ} \mathrm{C}\right)$ until transfer on dry ice to Metanomics Health, where they were stored $\left(-80^{\circ} \mathrm{C}\right)$ until analysis.

\section{Metabolite profiling}

We applied three types of MS analysis to all samples: GC-MS and liquid chromatography (LC)-MS/MS were used for broad profiling ${ }^{(8)}$, and solid-phase extraction-LC-MS/MS was used to determine catecholamine and steroid levels. Fractionation and derivatisation of samples and detection technologies for GC-MS and LC-MS/MS have been described previously ${ }^{(9)}$.
Steroids, catecholamines and their metabolites were measured by online solid-phase extraction-LC-MS/MS ${ }^{(10)}$. Absolute quantification was performed by means of stable isotopelabelled standards.

For GC-MS and LC-MS/MS profiling, data were normalised to the median of reference samples, which were derived from a pool formed from aliquots of all samples from that species to account for inter- and intra-instrumental variation. Pool samples were run in parallel through the whole process.

\section{Dataset normalisation and statistical methods}

Before statistical analysis, log transformation of ratios was conducted to assure normal distribution of data. The within- and between-animal variability was investigated by principal component analysis (PCA) of all peaks consistently present in the samples (consisting of metabolites with a known structure and metabolites with an unknown structure). PCA was performed using SIMCA-P version 10.0 (Umetrics AB, Umeå, Sweden) and the non-linear iterative partial least squares algorithm, an algorithm that works with missing data if they are relatively randomly distributed. Principal components (PC) 1-14 were analysed to examine whether the variation explained was associated with other metadata such as sampling day, feeding group, age or sibling group. A variance component analysis was then performed on each metabolite individually. The absolute and percentage of variance due to within- and between-animal variability was calculated and compared across metabolites.

\section{Results}

The individual is a major driver of variance in plasma metabolite profiles of both cats and dogs

In the present study, a total of 219 plasma metabolites for cats and 216 plasma metabolites for dogs were obtained at sufficient levels to allow semi-quantitative analysis. These included 152 (cats) and 150 (dogs) known, chemically identified metabolites, and sixty-seven (cats) and sixty-six (dogs) analytes that were consistently present but remained chemically unidentified. Of the total number of metabolites, 209 were present in both species.

In general, for each species, the samples from the same individual were clustered in PCA (see Fig. 1 as an example). This indicates that fasting plasma samples are reproducible and representative of the individual. In cats, PC1 explained $22 \%$ of the variability and PC2 explained 9\% of the variability. The PCA score plots (PC 1-14) were not clustered by feeding group, cat lodge, age, years spent neutered, blood sampling data or days on diet. In dogs, PC1 explained $16 \%$ of the variability and PC2 explained 9\% of the variability. The PCA score plots (PC 1-14) were not clustered by feeding group, dog unit, age, years spent neutered, blood sampling data, days on diet nor other data only relevant for dogs (e.g. toothbrushing regimen, pen-mate trial information). A trend was observed for a sibling group/parent effect in lower PC, but the study was not designed to investigate its significance. 


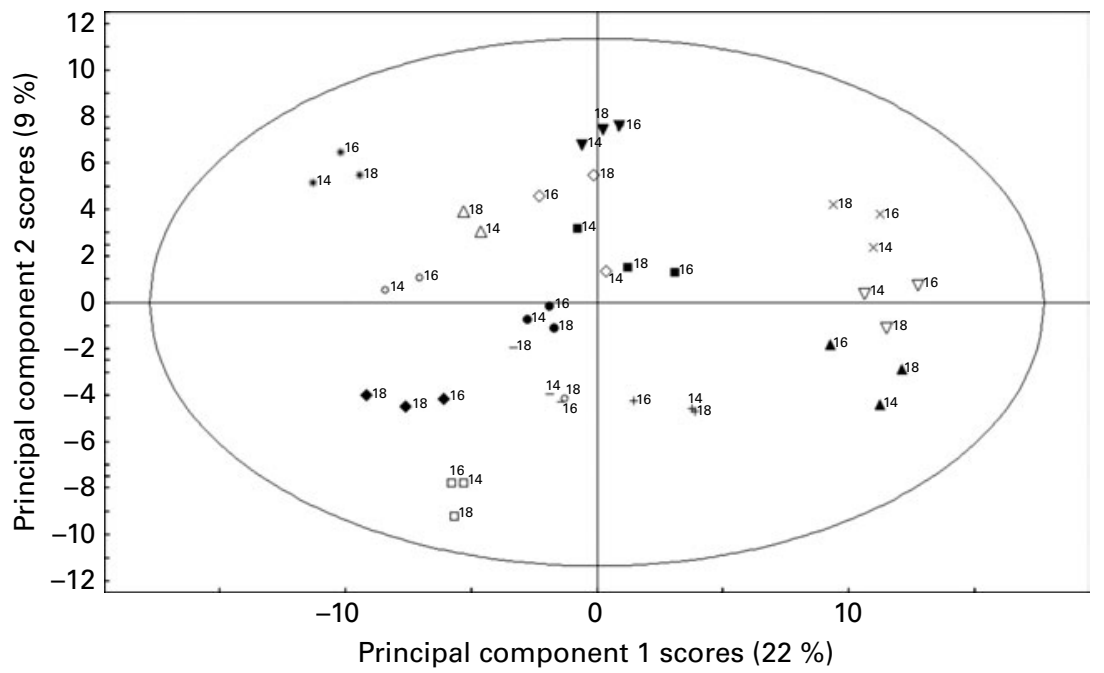

Fig. 1. Principal component analysis of the cat plasma metabolome. Samples from each cat are represented by a different symbol ${ }^{\star}, \Delta, \diamond, \bigcirc, \boldsymbol{\square}, \mathbf{\nabla}, \times,+,-, \square$, $\bullet, \boldsymbol{\Lambda})$. The number associated with each symbol is the sample day of the study. No sample was taken on day 16 for one cat $(\triangle)$.

\section{Many metabolites with inter-individual variance are common to both cats and dogs}

Having determined that the individual was a major driver of variance, the second objective was to identify metabolites that associated with the individual variation observed. The proportion of intra- and inter-individual variance was calculated for metabolites with a low intra-individual variance (SD < 0.05) (109 and 101 for cats and dogs, respectively). A total of fifteen cat and six dog metabolites had inter-individual variance accounting for at least $90 \%$ of the total variance, of which four metabolites were common to both species (campesterol, DHA, a cholestenol and a sphingosine moiety).

The majority of metabolites with a high inter-individual variance were primarily lipids, including sterols from the diet (campesterol), storage lipids (stearic acid, palmitic acid and oleic acid), structural fatty acids (DHA), lipids from cholesterol synthesis (cholestenol) and also sphingolipid-associated lipids (nervonic acid, erythrosphingosine, threosphingosine, 3-Omethylsphingosine and 5-O-methylsphingosine). Pairwise correlations between the fourteen fatty acid metabolites with $>90 \%$ inter-individual variance from cats had values $>0.583$ (mean 0.753). In dogs, the six fatty acids with $>90 \%$ interindividual variance had correlation values $>0.271$ (mean 0.598). Furthermore, many metabolites with $>75 \%$ inter-individual variance were common to both species and to similar areas of metabolism.

\section{Discussion}

Nutritional metabolomics may be a useful approach to investigate the relationship between the individual and their diet. In human subjects, the individual has been identified as a major cause of variance in nutritional metabolomic studies ${ }^{(11)}$, which confounds interpretation of data across a cohort. Genetics, sex, age, gastrointestinal flora and lifestyle differences are likely to be important factors, and some studies have attempted to compensate for these ${ }^{(12)}$, but there are logistic, ethical and economic constraints of feeding the same diet for a period of time to a group of individuals. A number of recommendations have been suggested to improve the design of nutritional metabolomics studies including controlling the age, sex, diet, lifestyle as well as time of sampling, storage and processing $^{(11)}$. Within companion animal nutritional studies, it is possible to run longer-term nutritional studies in controlled environments with reduced environmental variation and consistent sample handling. The present study design controlled for sex, age, breed and diet, and the triplicate samples per individual allowed analysis of intra- and inter-individual variance. The study also provided an assessment of reproducibility between trials, as the study was split into two trials sampled 4 weeks apart.

Using unsupervised multivariate methods (PCA), the individual was found to be a major driver of variance in the plasma metabolome. While the study was not designed to investigate the effect of age or sibling group, no significant effect was found for this or feeding group or number of days on diet. The latter may also indicate that a 2 -week feeding period is a suitable adaptation period to provide a consistent fasted plasma metabolome profile in both cats and dogs.

It was considered appropriate to identify metabolites with a high proportion of inter-individual variance, as it may identify specific metabolic processes that are tightly regulated by the individual and also indicate metabolites that may have cryptic responses to treatment effects in nutritional metabolomics studies. To allow identification of mutually correlated metabolites, a univariate approach was taken. More than $60 \%$ of the metabolites analysed in both cats and dogs had a higher proportion of inter-individual variance than that of intra-individual variance. Metabolites with a high proportion of interindividual variation were also generally consistent between species and correlated with each other. As they correlated with dietary-derived campesterol, this may indicate a diet effect. However, individual variance in these metabolites, including campesterol, did not correlate with dietary intake 
(either as $\mathrm{g}$ eaten or as $\mathrm{g}$ eaten/body weight $\mathrm{kg}^{0.75}$ ). The gastrointestinal flora is often cited as a main driver of urinary metabolite variance and has been suggested as a driver of variance in the plasma metabolome ${ }^{(2)}$. However, alternative explanations for the observed dominance of fats showing most inter-individual variance include differential fat transport from the gut and differential mobilisation of fat stores from adipocytes or myocytes during the fasting phase.

The small intestine is responsible for the transport of dietary and biliary cholesterol. Dogs are known to absorb dietary plant sterols and both dietary and biliary cholesterol efficiently through an ezetimibe-sensitive transporter, the Niemann-Pick C1-like 1 protein $^{(13)}$. This protein is essential for the uptake of cholesterol by enterocytes in the jejunum. In human subjects and mice, the enterocytes also express export transporters (ABCG5/ABCG8), and the presence of both import and export systems enables fine regulatory control of cholesterol levels ${ }^{(13)}$. Although speculative, if these efflux systems are also present in both cats and dogs, it may account for the relatively high proportion of inter-individual variance for these molecules.

Lipids are cellular membrane constituents, energy sources, involved in biological processes such as cell signalling processes and are implicated in a variety of diseases ${ }^{(3)}$. As such, the lipidome has been well studied, and its regulation is complex, dynamic and tightly controlled ${ }^{(3)}$. Although the data in the present study are a snapshot taken in the fasted state, it suggests that the regulation of some plasma lipids is specific to the individual even in a well-controlled study design. This feature may indicate that a cross-over design ought to be a major consideration in future nutritional study designs, and that interpretation of lipidomics data without a cross-over design requires particular caution.

In summary, the individual is an important driver of variance in the fasted plasma metabolome, and specific metabolites and areas of metabolism may be differentially regulated by individuals in two companion animal species.

\section{Acknowledgements}

None of the authors has a conflict of interest. The study was funded by the Waltham Centre for Pet Nutrition. A. C. performed the statistical analysis and helped in writing the manuscript; M. S. G. conducted the study; B. K. and D. R. designed and conducted the study, and helped in writing the manuscript; D. A. designed the study, helped in conducting the study, analysed the data and wrote the manuscript.

\section{References}

1. Kell BB \& Oliver SG (2004) Here is the evidence, now what is the hypothesis? The complementary roles of inductive and hypothesis-driven science in the post-genomic era. BioEssays 26, 99-105.

2. Gibney MJ, Walsh M, Brennan L, et al. (2005) Metabolomics in human nutrition: opportunities and challenges. Am J Clin Nutr 82, 497-503.

3. Oresic M (2009) Metabolomics, a novel tool for studies of nutrition, metabolism and lipid dysfunction. Nutr Metab Cardiovasc Dis 19, 816-824.

4. The Voice of the Pet Food Industry. http://www petfoodinstitute.org/ (accessed September 2010).

5. The European Pet Food Industry - Facts and Figs. http:// www.fediaf.org/the-european-pet-food-industry/facts-figures/ (accessed September 2010).

6. Viant MR, Ludwig C, Rhodes S, et al. (2007) Validation of a urine metabolome fingerprint in dog for phenotypic classification. Metabolomics 3, 453-463.

7. Beckman M, Enot DP, Overy DP, et al. (2010) Metabolite fingerprinting of urine suggests breed-specific dietary metabolism differences in domestic dogs. Br J Nutr 103, $1127-1138$.

8. van Ravenzwaay B, Cunha GC, Leibold E, et al. (2007) The use of metabolomics for the discovery of new biomarkers of effect. Toxicol Lett 172, 21-28.

9. Mutch DM, Fuhrmann JC, Rein D, et al. (2009) Metabolite profiling identifies candidate markers reflecting the clinical adaptation associated with Roux-en-Y gastric bypass surgery. PLoS One 4, e7905.

10. Yamada H, Yamahara A, Yasuda S, et al. (2002) Dansyl chloride derivatization of methamphetamine: a method with advantages for screening and analysis of methamphetamine in urine. J Anal Toxicol 26, 17-22.

11. Scalbert A, Brennan L, Fiehn O, et al. (2009) Massspectrometry-based metabolomics: limitations and recommendations for future progress with particular focus on nutrition research. Metabolomics 5, 435-458.

12. Walsh MC, Brennan L, Malthouse JPG, et al. (2006) Effect of acute dietary standardisation on the urinary, plasma, and salivary metabolomic profiles of healthy humans. $A m J$ Clin Nutr 84, 531-539.

13. Davis HR, Pula KK, Alton KB, et al. (2001) The synergistic hypocholesterolemic activity of the potent cholesterol absorption inhibitor, ezetimibe, in combination with 3-hydroxy-3-methylglutaryl coenzyme a reductase inhibitors in dogs. Metabolism 50, 1234-1241. 